\section{SP1-49 CERVICAL CANCER IN RELATION TO TOBACCO HABITS}

doi:10.1136/jech.2011.142976n.26

${ }^{1} \mathrm{~A}$ Juneja, ${ }^{*}{ }^{1} \mathrm{~A}$ Pandey, ${ }^{2} \mathrm{~A}$ Sehgal. ${ }^{1}$ National Institute of Medical Statistics, ICMR, New Delhi, India; ${ }^{2}$ Institute of Cytology and Preventive Oncology, ICMR, Noida, Uttar Pradesh, India

Introduction Non-communicable diseases (eg, cancer) have become a high profile issue for healthcare delivery planners in India. In view of limited resources, there is a need for an integrated approach to the control of cancers with common aetiologies. Cancer of the uterine cervix is one of the leading malignancies in Indian women. The present exercise attempts to study the relationship between cervical cancer and tobacco related cancers based on the age adjusted incidence rates as generated from reports of National Cancer Registry Programme of ICMR.

Methods Correlation analysis between the age adjusted rates of cancer of uterine cervix and tobacco related cancers such as mouth, oesophagus, lung were conducted for the three population based cancer registries at Bangalore, Mumbai and Chennai. Multivariate analysis was used for the relationship between cervical cancer with smoking habits based on the data generated through the ICMR study.

Results Results revealed that adjusted OR associated with development of cervical cancer among smokers was found to be of the order of 4 . There was a strong relationship between trends in cervical cancer with oral cancer and oesophageal cancer ranging from 0.4 to $0.8(p<0.05)$ substantiating the fact of a common aetiology. Conclusion The tobacco control programs which are a priority with government of India's National Cancer Control Program could also target cervical cancer control.

\section{SP1-50 CURRENT STATUS UPDATE ON NON-COMMUNICABLE DISEASES IN BANGLADESH}

doi:10.1136/jech.2011.142976n.27

${ }^{1} \mathrm{~S}$ Talukder, ${ }^{1} \mathrm{~S}$ H Khan, ${ }^{2} \mathrm{~N}$ Karim. ${ }^{1}$ Eminence, Dhaka, Bangladesh; ${ }^{2}$ WHO, Dhaka, Bangladesh

Background There is increasing evidence to suggest that the epidemiologic transition is well underway in Bangladesh and many of the low and middle income countries are facing a dual burden, with a huge load of infectious diseases and an increasing burden due to NCDs.

Objectives The objective of this paper is to describe the current status of NCDs in Bangladesh and policy guidelines in regarding the issue.

Methods This paper has been prepared based on literature review and content analysis. Relevant full articles (both academic and popular), abstracts and reports within the context of Bangladesh were reviewed from relevant journals.

Results In Bangladesh around $12.5 \%$ of all deaths are caused due to various types of cardiovascular diseases among $27.6 \%$ death due to NCDs. The prevalence of hypertension is reported as around $12 \%$ and the prevalence of diabetes in urban area is double $(10 \%)$ than rural area (5\%). The prevalence of COPD ( $\geq 30$ years) is $3 \%$ among general population and $6 \%$ for inpatients of medical college. Last 5 years government of Bangladesh has spent 950.07 lac BDT through HNPSP for NCD, in which arsenic program got more than $70 \%$ of the budget and WHO 5.53 lac USD for last 2 years to develop different policy, guideline and risk factor survey.

Conclusions As a developing country, in Bangladesh, addressing NCDs happens to be a multifaceted challenge. Appropriate strategies under high level political commitment and necessary funding as a part of the integrated development and health agenda of Bangladesh are essential.
SP1-51 DIABETES MELLITUS, GLUCOSE INTOLERANCE AND THE RISK OF CARDIOVASCULAR DISEASES: THE JAPAN ATHEROSCLEROSIS LONGITUDINAL STUDY-EXISTING COHORTS COMBINE (JALS-ECC)

doi:10.1136/jech.2011.142976n.28

A Kadota, ${ }^{*}$ K Miura, T Shinozaki, S Saitoh, Y Kiyohara, H Adachi, H Kawano, T Momotsu, H Amano, T Onoda, T Ando, M Taguri, A Harada, Y Ohashi, H Ueshima. The Japan Arteriosclerosis Longitudinal Study (JALS) Group, Tokyo, Japan

Objective To clarify the relationship of diabetes mellitus (DM) and impaired glucose tolerance (IGT) to the incidence of cardiovascular diseases (CVD) in Japanese general population.

Methods The Japan Arteriosclerosis Longitudinal Study Group conducted a meta-analysis of 20 cohort studies in Japan. We analysed a total of 42427 general Japanese men and women with the information of glucose tolerance (serum glucose, haemoglobin A1c, and/or treatment for diabetes). The HRs and 95\% CIs of CVD incidence (stroke or myocardial infarction) were estimated for DM and IGT using Poisson regression models.

Results The prevalence of DM was $7.1 \%$ and that of IGT was $13.8 \%$. During the mean follow-up of 8.1 years, 247 incidence of myocardial infarction and 999 incidence of stroke were confirmed. The multivariate adjusted HRs (95\% CIs) of CVD were 1.47 (1.20 to 1.80) for DM and 1.07 (0.90 to 1.27 ) for IGT. The multivariate adjusted HRs of myocardial infarction incidence were 1.49 (1.02 to 2.19) for DM and 0.83 (0.58 to 1.20$)$ for IGT. The multivariate adjusted HRs of stroke were 1.43 (1.18 to 1.73 ) for DM and 1.11 (0.93 to 1.32 ) for IGT. DM also increased the risk of ischaemic stroke (the HR was 1.78 [1.39 to 2.27]); however, we did not find any significant relationships of DM to hemorrhagic stroke and subarachnoid haemorrhage.

Conclusion This large scale meta-analysis of Japanese confirmed that DM increased the incident risk of CVD, especially ischaemic stroke and myocardial infarction. IGT also tended to increase the risk of CVD incidence, although the risk was not statistically significant.

\section{SP1-52 PREVALENCE OF FRAILTY IN ELDERS LIVING IN LONG-STAY INSTITUTIONS IN BRAZIL: CORRELATIONS AMONG FOUR INSTRUMENTS OF EVALUATION}

doi:10.1136/jech.2011.142976n.29

L M Santiago,* I E Mattos, L L Luz. Oswaldo Cruz Foundation, National School of Public Health, Rio de Janeiro, Brazil

Introduction The term frailty characterises elders at higher risk for the occurrence of adverse health outcomes in situations of environmental stress. We still do not have an assessment instrument that could be considered as the gold standard for this condition. This study aims to estimate the prevalence of frailty in older individuals living at long-stay institutions, through different instruments, and to evaluate their performance in this setting.

Methods This is a study with elderly residents of long-stay institutions in four Brazilian cities. The prevalence of frailty was estimated with four instruments: Fried phenotype (FP), Frailty Index based on Geriatric Status Scale (GSS-FI), Frail scale (FS) and Edmonton Frail Scale (EFS). The main characteristics of frail individuals according to each instrument were described. Correlations between the categories obtained with each instrument were analysed with Phi's correlation coefficient.

Results These are preliminary results for 340 elders. The estimated prevalence of frailty was $54.3 \%$ for FP, $11.3 \%$ for GSS-FI, $12.1 \%$ for FS and $36.1 \%$ for EFS. The mean age of frail individuals varied from 72 to 75 years, depending on the assessment instrument. Frailty predominated in males $(60.0-80.0 \%), 4$ or less years of schooling (76.0-91.0\%), single (44.0-59.0\%) and time in the institution of up 
to 10 years $(64.0-87.0 \%)$. The correlation coefficients varied from 0.1 to $0.4(\mathrm{p}<0.05)$.

Conclusions Although most of the correlations between the instruments were moderate, frailty prevalence varied largely according to the instrument used to assess the condition. These differences are justified because of the different domains assessed by each instrument.

\section{SP1-53 THE EFFECT OF SOCIAL DEPRIVATION ON WEIGHT IN THE UK CYSTIC FIBROSIS POPULATION}

doi:10.1136/jech.2011.142976n.30

${ }^{1} \mathrm{D}$ Taylor-Robinson, ${ }^{*} \mathrm{M}$ Whitehead, ${ }^{2} \mathrm{P}$ Diggle, ${ }^{1} \mathrm{R}$ Smyth. 'University of Liverpool, Liverpool, UK; ${ }^{2}$ Lancaster University, Lancaster, UK

Background Maintaining nutritional status is a key component of care in people with cystic fibrosis. Low socioeconomic status has been linked with poor outcomes in CF. We explored, for the first time in a UK-wide cohort, longitudinal weight gain and its relationship with socioeconomic status (SES).

Methods We undertook a retrospective longitudinal cohort study of 4346 people with cystic fibrosis aged $<20$ years $(21132$ observations) in UK CF registry between 1995 and 2006. Census based indices of multiple deprivation (IMD) from the UK constituent counties were used as small area measures of SES. Piecewise mixed model regression was used to estimate the effect of SES on weight-for-age z-score (WFA).

Results WFA was significantly lower in the most deprived quintile at all time points. The estimated WFA at birth (intercept) was -0.64 in the least deprived quintile compared to -1.31 in the most deprived (mean difference $0.6795 \%$ CI 0.42 to 0.92 ). The population WFA increased up to age three by 0.2 per year, and then declined subsequently by -0.033 per year. There was a significantly steeper improvement in WFA in the most deprived quintile in the first 3 years (mean difference per year $0.1395 \%$ CI 0.06 to 0.20 ), with no difference in the rate of decline subsequently.

Conclusions Social deprivation is associated with lower WFA in the UK cystic fibrosis population, but there is a period of increased weight gain in the first 3 years, highlighting the importance of early diagnosis and treatment.

\section{SP1-54 RESULTS OF A 3-YEAR WORKPLACE WELLNESS PROGRAM AMONG A WORK COHORT IN KUALA LUMPUR, MALAYSIA}

doi:10.1136/jech.2011.142976n.31

${ }^{1,2} \mathrm{~F}$ M Moy, ${ }^{* 1}$ A M Bulgiba. ${ }^{1}$ Faculty of Medicine, University of Malaya, Kuala Lumpur, Malaysia; ${ }^{2} J u l i u s$ Centre University of Malaya, Kuala Lumpur, Malaysia

Introduction Workplace wellness programs are proven to be an important strategy to prevent cardiovascular diseases and stroke. Therefore, a workplace wellness program was conducted among a work cohort in Kuala Lumpur with the aim of improving risk factors for cardiovascular disease and stroke.

Methods This was an open cohort where all employees aged 35 years and above in the workplace were invited to participate in the wellness program. This program involved voluntary yearly screening with results dissemination, lifestyle counselling or referral for medical treatment when necessary. Ethics clearance and informed consent were obtained. Fasting blood glucose, lipid profile, blood pressure, weight, height and waist circumference were measured following standard protocols. This paper will report the findings over a period of 3 years (2008-2010).

Results Majority of this cohort $(\mathrm{n}=1923)$ were Malays $(77.9 \%)$, followed by Chinese (8.4\%), Indians $(9.9 \%)$ and others $(3.8 \%)$. The proportion of female participants was $53 \%$. The mean age of this cohort was $48.5+5.2$ years at baseline. In the General Linear Model after adjusted for race and sex, LDL cholesterol and total cholesterol levels were significantly improved after 3 years. Their mean $(95 \% \mathrm{CI})$ total cholesterol levels over the 3 years $(2008-2010)$ were $5.59(95 \%$ CI 5.49 to 5.69 ), 5.47 (5.40 to 5.55 ) and 5.39 (5.32 to 5.47 ) $\mathrm{mmol} / \mathrm{l}$ respectively. The mean LDL cholesterol levels were 3.59 (3.52 to 3.66), 3.49 (3.43 to 3.26 ) and 3.33 (3.56 to 3.40 ) $\mathrm{mmol} / \mathrm{l}$ respectively. Conclusions Our findings show that low intensity workplace wellness program is effective in improving some cardiovascular risk factors.

\section{SP1-55 WEOAYA: A WHOLE POPULATION CARDIOVASCULAR SCREENING PROGRAMME IN ABU DHABI, UNITED ARAB EMIRATES}

doi:10.1136/jech.2011.142976n.32

C Hajat, ${ }^{*} 0$ Harrison, Z Al Siksek. Department of Public Health \& Research, Health Authority Abu Dhabi, Abu Dhabi, United Arab Emirates

Introduction Data have shown the UAE to have high rates of cardiovascular disease but the risk factor burden remained poorly studied. This study describes the baseline cardiovascular risk profile of the National population of Abu Dhabi.

Methods Adults aged 18 years or over were screened using selfreported indicators, anthropometric measures and blood tests in primary care.

Results The study included 50138 subjects. Mean age (SD) was 36.82 (14.30) years with 21663 (43\%) males and 28474 (57\%) females. Numbers and crude prevalence rates were for obesity 17556 (35\%), overweight 15823 (32\%), central obesity 27480 (55\%), diabetes 8528 (18\%), pre-diabetes 13127 (27\%), dyslipidaemia 21655 (44\%) and hypertension 11377 (23.1\%). Smoking rates were $5570(26 \%)$ in males and $221(0.8 \%)$ in females. Age-standardised rates for diabetes and pre-diabetes were 11792 (25\%) and 14158 (30\%), obesity and overweight were 19711 (41\%) and 16298 (34\%). Family history of premature cardiovascular disease was independently associated with a past history of cardiovascular disease with an OR of 5.34 (95\% CI 3.79 to 7.52 ).

Conclusion This population-wide cardiovascular screening programme in the Middle East has demonstrated a very high cardiovascular burden for this small and young population. The data form a baseline against which progress is monitored for the population-wide Abu Dhabi Cardiovascular Disease Programme.

\section{SP1-56 ASSOCIATION BETWEEN OVERWEIGHT, OBESITY AND SELF-PERCEPTION OF BODY WEIGHT IN ADULTS}

doi:10.1136/jech.2011.142976n.33

${ }^{1} \mathrm{G}$ França, ${ }^{*} \mathrm{D}$ Gigante, ${ }^{2} \mathrm{M}$ T Olinto. ${ }^{1}$ Universidade Federal de Pelotas, Pelotas, Rio Grande do Sul, Brazil; ${ }^{2}$ Universidade do Vale do Rio dos Sinos, Rio Grande do Sul, Brazil

Introduction This study aimed to examine the association between overweight, obesity and self-perception of body weight in adults.

Methods A cross-sectional population-based study was carried out in the city of Pelotas, southern Brazil, with a sample of individuals aged 20-59 years. Weight and height of the participants were measured by previously trained evaluators. Overweight and obesity were defined as body mass index $\geq 25 \mathrm{~kg} / \mathrm{m}^{2}$ and $\geq 30 \mathrm{~kg} / \mathrm{m}^{2}$, respectively. Self-perceived body weight status was directly and indirectly assessed. The participants were first asked whether they perceive themselves as too thin, thin, normal, fat or too fat and then the difference between reported ideal and actual body weight measured after the interview was calculated. 